\title{
MONOTONICALLY NORMAL SPACES
}

BY

\author{
R. W. HEATH( $\left.{ }^{1}\right)$, D. J. LUTZER( $\left.{ }^{1}\right)$, AND P. L. ZENOR
}

ABSTRACT. This paper begins the study of monotone normality, a common property of linearly ordered spaces and of Borges' stratifiable spaces. The concept of monotone normality is used to give necessary and sufficient conditions for stratifiability of a $T_{1}$-space, to give a new metrization theorem for $p$-spaces with $G_{\delta}$-diagonals, and to provide an easy proof of a metrization theorem due to Treybig. The paper concludes with a list of examples which relate monotone normality to certain familiar topological properties.

1. Introduction. The property of monotone normality first appears, without name, in Lemma 2.1 of C. R. Borges' paper On stratifiable spaces [5]. In [29], $P$. Zenor named the property and announced results relating monotone normality to metrizability and stratifiability. Subsequently Heath and Lutzer ([17], [18]) and Borges [7] announced results complementary to Zenor's original work, showing, in particular, that monotone normality unexpectedly holds in one large class of spaces-the linearly ordered spaces-but fails to hold in others where it might be expected.

This paper is a combination of the independent studies conducted by Zenor and by Heath and Lutzer. $\$ \$ 2$ through 4 are the work of Zenor, with the exception of Lemma 2.2 which, together with $\$ \$ 5$ through 7 , is due to Heath and Lutzer. The authors would like to thank the editor for suggesting this format.

2. Definitions and preliminary results. Throughout this paper all spaces are assumed to be at least $T_{1}$ and "mapping" means "continuous onto function." The set of natural numbers is denoted by the letter $N$.

2.1. Definition. A space $X$ is monotonically normal if there is a function $G$ which assigns to each ordered pair $(H, K)$ of disjoint closed subsets of $X$ an open set $G(H, K)$ such that

(a) $H \subset G(H, K) \subset G(H, K)^{-} \subset X \backslash K$;

(b) if $\left(H^{\prime}, K^{\prime}\right)$ is a pair of disjoint closed sets having $H \subset H^{\prime}$ and $K \supset K^{\prime}$

Received by the editors October 15, 1971 and, in revised form, May 25, 1972.

AMS (MOS) subject classifications (1970). Primary 54D15, 54D20; Secondary 54E20, 54E35, 54F05.

Key words and phrases. Monotone normality, collectionwise normality, stratifiable

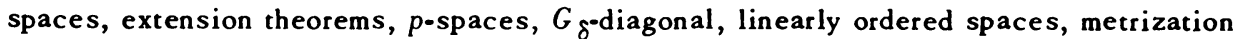
theorems, $\beta N$.

(1) Partially supported by NSF Grant GP 29401. 
then $G(H, K) \subset G\left(H^{\prime}, K^{\prime}\right)$.

The function $G$ is called a monotone normality operator for $X$.

2.2. Lemma. Any monotonically normal space has a monotone normality operator $G$ satisfying $G(A, B) \cap G(B, A)=\varnothing$ for any pair $(A, B)$ of disjoint closed sets. Furthermore, each of the following properties is equivalent to monotone normality of a space $X$ :

(a) There is a function $G$ which assigns to each ordered pair $(S, T)$ of separated(2) subsets of $X$ an open set $G(S, T)$ satisfying

(i) $S \subset G(S, T) \subset G(S, T)^{-} \subset X \backslash T$;

(ii) if $\left(S^{\prime}, T^{\prime}\right)$ is a pair of separated sets baving $S \subset S^{\prime}$ and $T \supset T^{\prime}$ then $G(S, T) \subset G\left(S^{\prime}, T^{\prime}\right)$.

(b) There is a function $H$ which assigns to each ordered pair $(p, C)$, with $C$ closed and $p \in X \backslash C$, an open set $H(p, C)$ satisfying:

(i) $p \in H(p, C) \subset X \backslash C$;

(ii) if $D$ is closed and $p \notin C \supset D$ then $H(p, C) \subset H(p, D)$;

(iii) if $p \neq q$ are points of $X$ then $H(p,\{q\}) \cap H(q,\{p\}) \doteq \varnothing$.

Proof. To prove the first statement, let $G^{\prime}$ be any monotone normality operator for $X$ and define $G(A, B)=G^{\prime}(A, B) \backslash G^{\prime}(B, A)^{-}$. Then $G$ is a monotone normality operator for $X$ having $G(A, B) \cap G(B, A)=\varnothing$.

Clearly $(a) \Rightarrow$ (monotone normality) $\Rightarrow(b)$. We show that (b) implies (a). For any pair $(S, T)$ of separated sets let

$$
G(S, T)=\bigcup\left\{H\left(p, T^{-}\right) \mid p \in S\right\} .
$$

Clearly $S \subset G(S, T)$; and $G(S, T)^{-} \subset X \backslash T$ since for each $q \in T$, the set $H\left(q, S^{-}\right)$ is a neighborhood of $q$ disjoint from $G(S, T)$. Property (ii) of (a) follows directly from (ii) of (b).

2.3. Remarks. (a) The property described in 2.2 (a) was originally called complete monotone normality [29].

(b) The equivalence of statement (a) with monotone normality was obtained independently by Borges [7] who has given several other properties equivalent to monotone normality in [8].

(c) Lemma 2.2 makes it clear that monotone normality is a hereditary property.

2.4. Definition. A space $(X, \mathfrak{J})$ is semistratifiable [12] if there is a function $S$ : \{closed subsets of $X\} \times N \rightarrow \mathcal{T}$, called a semistratification of $X$, such that:

(a) If $H \subset K$ are closed subsets of $X$ then $S(H, n) \subset S(K, n)$ for each $n \in N$;

(b) $H=\bigcap\{S(H, n) \mid n \in N\}$ for each closed set $H \subset X$.

(2) Two subsets $H, K$ of $X$ are separated if $H \cap K^{-}=\varnothing=K^{-} \cap H^{-}$. 
If, in addition, the function $S$ satisfies

(c) $H=\bigcap\left\{S(H, n)^{-} \mid n \in N\right\}$ for each closed set $H \subset X$, then $X$ is stratifiable [5] and $S$ is called a stratification of $X$.

2.5. Theorem. A space $X$ is stratifiable if and only if $X$ is semistratifiable and monotonically normal.

Proof. Suppose $X$ admits a monotone normality operator $G$ and a semistratification $T$. For each closed set $K \subset X$ and $n \in N$ let $S(K, n)=G(K, X \backslash T(K, n))$. Then $S$ is a stratification of $X$. The converse follows from a result of Borges [5, Lemma 2.1] and Definition 2.4.

2.6. Theorem. Suppose $f: X \rightarrow Y$ is a closed mapping. If $X$ is monotonically normal, so is $Y$.

Proof. Let $G$ be a monotone normality operator for $X$. For disjoint closed subsets $H, K$ of $Y$, let

$$
\dot{G}^{\prime}(H, K)=Y \backslash \gamma\left[X \backslash G\left(f^{-1}(H), f^{-1}(K)\right)\right] .
$$

Then $G^{\prime}$ is a monotone normality operator for $Y$.

It follows immediately from (2.6) that if $X$ can be covered by a locally finite (or even hereditarily closure preserving (3)) collection of closed monotonically normal subspaces, then $X$ is monotonically normal. It might be interesting to know whether $X$ must be monotonically normal provided that $X$ is dominated (cf. [5, Definition 7.1]) by a collection of such subspaces.

3. Special properties of monotonically normal spaces. Recall that a space $X$ is collectionwise normal [3] if for each discrete collection(4) $\mathfrak{H}$ of closed subsets of $X$ there is a disjoint collection $\mathfrak{X}=\{D(H) \mid H \in \mathcal{H}\}$ of open subsets of $X$ with the property that $H \subset D(H)$ for each $H \in \mathcal{H}$. It is easily seen that $\mathfrak{D}$ may be taken to be a discrete collection of open sets.

3.1. Theorem. Any monotonically normal space is (bereditarily) collectionwise normal.

Proof. Let $G$ be a monotone normality operator for $X$ which satisfies $G(A, B) \cap G(B, A)=\varnothing$ for each pair $(A, B)$ of disjoint closed sets in $X$ (cf. Lemma 2.2). Let $\mathcal{H}$ be a discrete family of closed subsets of $X$. For each $H \in \mathcal{H}$ define $D(H)=G\left(H, \bigcup\left\{H^{\prime} \in \mathcal{H} \mid H^{\prime} \neq H\right\}\right)$. If $H_{1} \neq H_{2}$ are members of $\mathcal{H}$ then $D\left(H_{1}\right) \subset G\left(H_{1}, H_{2}\right)$ and $D\left(H_{2}\right) \subset G\left(H_{2}, H_{1}\right)$ so that $D\left(H_{1}\right) \cap D\left(H_{2}\right) \subset G\left(H_{1}, H_{2}\right) \cap$ $G\left(H_{2}, H_{1}\right)=\varnothing$. Therefore $\mathscr{D}=\{D(H) \mid H \in \mathcal{H}\}$ is the required disjoint collection

(3) A collection $\left\{S_{i} \mid i \in I\right\}$ of subsets of $X$ is hereditarily closure preserving if

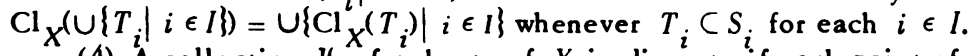

(4) A collection $H$ of subsets of $X$ is discrete if each point of $X$ has a neighborhood which meets at most one member of $K$. 
of open sets. Thus any monotonically normal space is collectionwise normal. Hereditary collectionwise normality follows from (2.3) (c).

3.2. Notation. For any space $Y, C(Y, I)$ denotes the set of all continuous functions from $Y$ to the unit interval $I=[0,1]$.

3.3. Theorem. Suppose $H$ is a closed subspace of a monotonically normal space $X$. Then there is a function $Q: C(H, I) \rightarrow C(X, I)$ sucb that:

(a) for eacb $f \in C(H, I), Q(f)$ extends f, i.e., $Q(f)(x)=f(x)$ for each $x \in H$;

(b) if $f, g \in C(H, I)$ and $f(x) \leq g(x)$ for every $x \in H$, then $Q(f)(x) \leq Q(g)(x)$ for eacb $x \in X$.

Proof. The construction is of the type used to prove Urysohn's Lemma. Let $D$ be a monotone normality operator for $X$. For each $f \in C(H, I)$ and for each real number of the form $p / 2^{q}$, where $-1 \leq p<2^{q}$ and $q>0$ are integers, we obtain an open set $D_{f}\left(p / 2^{q},(p+1) / 2^{q}\right)$ such that:

(1) $f^{-1}\left(\left[0, p / 2^{q}\right]\right) \subset D_{f}\left(p / 2^{q},(p+1) / 2^{q}\right) \subset D_{f}\left(p / 2^{q},(p+1) / 2^{q}\right)^{-} \subset$ $x \backslash f^{-1}\left(\left[(p+1) / 2^{q}, 1\right]\right)$;

(2) $D_{f}\left((p-1) / 2^{q}, p / 2^{q}\right)^{-} C D_{f}\left(p / 2^{q},(p+1) / 2^{q}\right)$;

(3) if $p$ is odd, then $D_{f}\left((p-1) / 2^{q},(p+1) / 2^{q}\right)^{-} \subset D_{f}\left(p / 2^{q},(p+1) / 2^{q}\right)$;

(4) if $p$ is even, then $D_{f}\left(p / 2^{q},(p+1) / 2^{q}\right)^{-} \subset D_{f}\left(p / 2^{q},(p+2) / 2^{q}\right)$; and

(5) if $f(x) \leq g(x)$ for each $x$ in $H$, then $D_{f}\left(p / 2^{q},(p+1) / 2^{q}\right) \subset$ $D_{g}\left(p / 2^{q},(p+1) / 2^{q}\right)$ for each $p / 2^{q}$.

To this end, let $f$ denote a member of $C(H, I)$. For all $q>0$, let

$$
D_{f}\left(-1 / 2^{q}, 0\right)=\varnothing \quad \text { and } \quad D_{f}\left(1,\left(2^{q}+1\right) / 2^{q}\right)=X \text {. }
$$

Let

$$
H_{f}(0,1 / 2)=f^{-1}(\{0\}), \quad K_{f}(0,1 / 2)=f^{-1}([1 / 2,1])
$$

and

$$
\begin{gathered}
D_{f}(0,1 / 2)=D\left(H_{f}(0,1 / 2), K_{f}(0,1 / 2)\right) \\
H_{f}(1 / 2,1)=f^{-1}([0,1 / 2]) \cup D_{f}(0,1 / 2)^{-}, \quad K_{f}(1 / 2,1)=f^{-1}(\{1\})
\end{gathered}
$$

and

$$
\nu_{f}(1 / 2,1)=D\left(H_{f}(1 / 2,1), K_{f}(1 / 2,1)\right) .
$$

In general let 
$H_{f}\left(n / 2^{q},(n+1) / 2^{q}\right)=f^{-1}\left(\left[0, n / 2^{q}\right]\right) \cup \begin{cases}D\left((n-1) / 2^{q},(n+1) / 2^{q}\right)^{-} & \text {if } n \text { is odd, } \\ D\left((n-1) / 2^{q}, n / 2^{q}\right)^{-} & \text {if } n \text { is even, }\end{cases}$ $K_{f}\left(n / 2^{q},(n+1) / 2^{q}\right)=f^{-1}\left(\left[(n+1) / 2^{q}, 1\right]\right) \cup\left[X \backslash\left\{\begin{array}{ll}D\left((n+1) / 2^{q},(n+3) / 2^{q}\right) & \text { if } n \text { is odd, } \\ D\left(n / 2^{q},(n+2) / 2^{q}\right) & \text { if } n \text { is even }\end{array}\right]\right.$ and

$$
D_{f}\left(n / 2^{q},(n+1) / 2^{q}\right)=D\left(H_{f}\left(n / 2^{q},(n+1) / 2^{q}\right), K_{f}\left(n / 2^{q},(n+1) / 2^{q}\right)\right) .
$$

Now, to obtain the function $Q$, let $f \in C(H, I)$. For each $x$ in $X$, define

$$
\mathcal{Q}(f)(x)=\operatorname{glb}\left\{p / 2^{q}: x \in D_{f}\left((p-1) / 2^{q}, p / 2^{q}\right)\right\} .
$$

It follows from (1) that for each $x$ in $H, Q(f)(x)=f(x)$. Also, it is clear from (5) that if for each $x$ in $H, f(x) \leq g(x)$, then for each $x$ in $X, Q(f)(x) \leq Q(g)(x)$. It remains to show that $Q(f)$ is in $C(X, I)$; that is, that each $Q(f)$ is continuous.

Before proceeding, note that it follows from (2), (3), and (4) that

(6) if $q>t$ and $D_{f}\left(p / 2^{q},(p+1) / 2^{q}\right)$ intersects $X \backslash D_{f}\left(s / 2^{t},(s+1) / 2^{t}\right)$, then $(s+1) / 2^{t} \geq(p+1) / 2^{q}$.

Now, let $x \in X$ and let $\epsilon>0$. Let $q$ be an integer such that $1 / 2^{q}<\epsilon / 2$. Let $p$ be the first integer such that $x$ is in $D\left((p-1) / 2^{q}, p / 2^{q}\right)$. It follows from (6) that $Q(f)(x) \geq(p-1) / 2^{q}$. Let

$$
U=D\left((p-1) / 2^{q}, p / 2^{q}\right) \backslash \operatorname{cl} D\left((2(p-1)-1) / 2^{q+1},(2(p-1)) / 2^{(q+1)}\right) .
$$

$U$ is an open set in $X$ that contains $x$. Let $y \in U$. Clearly, $Q(f)(y) \leq p / 2^{q} \leq$ $Q(f)(x)+\epsilon$. It follows from (6) that $Q(f)(y) \geq(2(p-1)-1) / 2^{(q+1)}$; and so, $Q(f)(x)-\epsilon<p / 2^{q}-1 / 2^{q}-1 / 2^{(q+1)}=(2(p+1)-1) / 2^{(q+1)} \leq Q(f)(y)$. Thus, $|Q(f)(y)-Q(f)(x)|<\epsilon$.

Remarks. (a) The authors do not know whether the converse of 3.3 is true. (b) In Theorem 4.3 of [5], Borges proved that if $H$ is a closed subspace of a stratifiable space $X$ and if $C(H)$ and $C(X)$ are the real vector spaces of continuous real-valued functions on $H$ and $X$ respectively, then there is a linear transformation $Q: C(H) \rightarrow C(X)$ which satisfies (a) and (b) of our Theorem 3.3. The authors do now know whether Borges' result can be proved for monotonically normal spaces.

4. Products of monotonically normal spaces. The reader should compare the techniques and results of this section with those of [19].

4.1. Theorem. If $X \times Y$ is monotonically normal then either no countable subset of $X$ bas a limit point or $Y$ is stratifiable. 
Proof. Suppose that $M^{\prime}=\left\{m_{n} \mid n \in N\right\}$ is a countable subset of $X$ having a limit point $p$. We may assume that $p \notin M^{\prime}$. Let $M=M^{\prime} \cup\{p\}$. Since $X \times Y$ is monotonically normal, so is the subspace $M \times Y$ (cf. 2.3(c)). Let $G$ be a monotone normality operator for $M \times Y$ as described in 2.2(a). (Thus $G(S, T)$ is defined for pairs of separated subsets of $M \times Y$.) For each closed set $F \subset Y$, the sets $H_{F}=\{(x, y) \in M \times Y \mid y \in F$ and $x \neq p\}$ and $K_{F}=\{(p, y) \in M \times Y \mid y \notin F\}$ are separated subsets of $M \times Y$. For each $n \in N$ let $T(F, n)=\left\{y \in Y \mid\left(m_{n}, y\right) \epsilon\right.$ $\left.G\left(H_{F}, K_{F}\right)\right\}$. It is easily seen that $T$ is a stratification for $Y$.

4.2. Corollary. The following properties of a space $X$ are equivalent:

(a) $X$ is stratifiable;

(b) $X \times M$ is monotonically normal for every metric space $M$;

(c) If $M_{0}=\{0\} \cup\{1 / n \mid n \in N\}$ bas its usual topology, then $X \times M_{0}$ is monotonically normal;

(d) $X^{\omega_{0}}$, the product of countably many copies of $X$, is monotonically normal.

Proof. Clearly (a) $\Rightarrow(\mathrm{b}) \Rightarrow$ (c) and (a) $\Rightarrow$ (d). We show that (d) $\Rightarrow$ (c) $\Rightarrow$ (a). If $X$ has at least two points, then $X^{\omega_{0}}$ contains a sequence converging to a limit point, i.e. $X$ contains a copy of $M_{0}$. Then $X \times M_{0}$ is a subspace of $X \times X^{\omega_{0}}$ $=X^{\omega_{0}}$ so that $X \times M_{0}$ is monotonically normal. Thus (d) $\Rightarrow$ (c). That (c) $\Rightarrow$ (a) follows directly from Theorem 4.1 .

Example 7.6 shows that there is a nonstratifiable space $X$ such that $X^{n}$. the product of $n$ copies of $X$, is monotonically normal for each $n \in N$.

4.3. Definition [1]. A completely regular space $X$ is a $p$-space if there is a sequence $\langle\mathcal{U}(n)\rangle$ of covers of $X$ by open subsets of $\beta X$ such that $\bigcap\left\{\mathrm{St}_{\mathrm{t}}(x, \mathcal{U}(n)) \mid\right.$ $n \in N\} \subset X$ for each $x \in X .(5)$

The definition of $p$-spaces will be needed in a later section. The definitions of $M$-spaces and $w \Delta$-spaces can be found in [25] and [6] respectively.

4.4. Corollary. A space $X$ is metrizable if and only if $X^{2}=X \times X$ is monotonically normal and $X$ satisfies one of the following conditions:

(a) $X$ is a p-space;

(b) $X$ is an M-space;

(c) $X$ is a $w \Delta$-space.

Proof. If $X$ is discrete then $X$ is metrizable. If $X$ has a limit point and satisfies (a), (b) or (c), then some countable subset of $X$ has a limit point. According to (4.1), $X$ is stratifiable. It is shown in [12] that a semistratifiable $p$-space is a Moore space (see also [9]); thus $X$ is metrizable. Any M-space is a $w \Delta$-space and it was proved in [6] that a stratifiable $w \Delta$-space is metrizable.

(5) If $U$ is a collection of subsets of $X$ and if $p \in X$, then $\operatorname{St}(p$, บ ) $=U\{U \in$ บ| $p \in U\}$. 


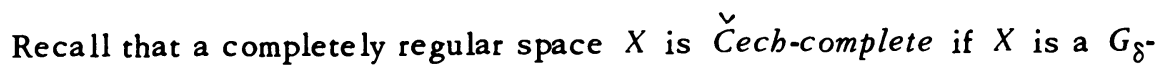
subset of $\beta X[13]$ and that a metrizable space is completely metrizable if and only if it is Čech-complete [10].

4.5. Corollary. A space $X$ is completely metrizable if and only if $X$ is Čech complete and $X \times X$ is monotonically normal.

4.6. Corollary. If $X$ is locally countably compact space sucb that $X^{2}=X \times$ $X$ is monotonically normal, then $X$ is metrizable.

Proof. If $X$ is not discrete then some countable subset of $X$ has a limit point. Thus $X$ is stratifiable. Since a stratifiable countably compact space is compact $([14],[12]), X$ is locally compact; hence $X$ is Čech-complete. Thus $X$ is metrizable.

5. Monotone normality in ordered spaces.

5.1. Definition. Let $(X,<)$ be a linearly ordered set. A subset $C \subset X$ is convex if $\{x \in X \mid a<x<b\}$ is a subset of $C$ whenever $a, b$ are points of $C$. Let $S$ be any subset of $X$. A convex component of $S$ is a subset $C \subset S$ which is maximal with respect to the property " $C \subset S$ and $C$ is convex."

Clearly, any subset of $X$ can be uniquely expressed as a union of its convex components and distinct convex components of a set are disjoint.

5.2. Definition. A linearly ordered topological space (abbreviated LOTS) is a triple $(X, g,<)$ where $<$ is a linear ordering of the set $X$ and where $g$ is the usual open-interval topology of $<$.

\subsection{Theorem. Any LOTS is monotonically normal.}

Proof. We verify that $X$ admits a function $G(p, C)$ as in $(2.2)(b)$. Let $W$ be any well-ordering of the set $X$. (The order $W$ need not have any relation to the linear ordering which defines the topology of $X$.$) For any pair (p, C)$ where $C$ is closed and $p \in X \backslash C$, let $I(p, C)$ be the convex component of $X \backslash C$ which contains tains $p$.

If $I_{-}(p, C)=\{y \in I(p, C) \mid y<p\} \neq \varnothing \quad$ let $x(p, C)$ be the W-first point of $I_{-}(p, C)$ and let $y(p, C)$ be the W-first point of $I_{+}(p, C)=\{y \in I(p, C) \mid y>p\}$ if $I_{+}(p, C) \neq \varnothing$. Now define

$$
G(p, C)= \begin{cases}]_{x}(p, C), y(p, C)[ & \text { if } I_{-}(p, C) \neq \varnothing \neq I_{+}(p, C) \\ {[p, y(p, C)[} & \text { if } I_{-}(p, C)=\varnothing \neq I_{+}(p, C) \\ ]_{x}(p, C), p\right] & \text { if } I_{-}(p, C) \neq \varnothing \neq I_{+}(p, C) \\ \{p\} & \text { if } I_{-}(p, C)=\varnothing=I_{+}(p, C)\end{cases}
$$

In any case, $G(p, C)$ is an open subset of $X$ and it is clear that $G(p, C)$ satisfies the three conditions of $(2.2)(b)$. 
Combining (5.3), (2.3)(c) and (3.1) we obtain an easy proof of the following:

5.4. Corollary [27]. Any LOTS is bereditarily collectionwise normal.

5.5. Definition. A generalized ordered space is a triple $(\dot{X}, \mathfrak{T},<)$ where $\mathfrak{T}$ is a topology on $X$ and $<$ is a linear ordering of $X$ such that

(a) $\mathscr{g}(<) \subset \mathcal{T}$ where $\mathscr{g}(<)$ denotes the usual open-interval topology of $<$;

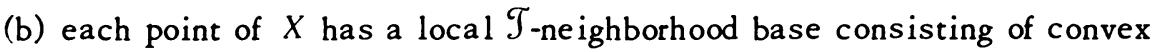
sets.

It is known that a space $X$ can be topologically embedded in some LOTS if and only if there is a linear ordering of $X$ making $X$ a generalized ordered space ([10], [20]). See Examples 7.1, 7.2, and 7.6.

5.6. Corollary. Any generalized ordered space is monotonically normal.

5.7. Questions. (a) There are several well-known progfs that any LOTS is normal ([4], [22], [27]), all of which invoke the axiom of choice. In [4], Birkhoff asks whether one can prove that any LOTS is normal without the axiom of choice. As far as the authors know, that question is still open (except, of course, for LOTS which are order-complete and order-bounded).

(b) What is the relation between monotone normality and $\boldsymbol{\kappa}_{0}$-full normality [22] (another property of any LOTS which implies collectionwise normality)?

Let us conclude this section with a simplified proof of a metrization theorem due to Treybig [28].

5.8. Corollary. Let $X$ and $Y$ be infinite Hausdorff spaces and suppose that $X \times Y$ is a continuous image of a compact LOTS L. Then $X$ and $Y$ are metrizable.

Proof. Let $f: L \rightarrow X \times Y$ be continuous and onto. Since $L$ is compact, $f$ is a closed map. According to (2.6), $X \times Y$ is monotonically normal. Furthermore $X \times Y$ is compact; thus $X$ and $Y$ each contain a countably infinite set with a limit point. According to (4.1), both $X$ and $Y$ are stratifiable. Being stratifiable and compact, $X$ and $Y$ are metrizable.

\section{Metrization of $p$-spaces.}

6.1. Definition. A space $X$ has a $G_{\delta}$-diagonal if the set $\Delta_{X}=\{(x, x): x \in X\}$ is a $G_{\delta}$-subset of $X \times X$.

It is known that $X$ has a $G_{\delta}$-diagonal if and only if there is a sequence $\left\langle G_{(n)}(n)\right.$ of open covers of $X$ such that $\bigcap\left\{\right.$ St $\left.\left(x, \varrho_{(n)}\right): n \in N\right\}=\{x\}$ for each $x \in X .(6)$

In proving our metrization theorem we use two results, one due to Bing and the other obtained by Creede.

(6) See footnote (5), p. 486. 
6.2. Theorem. (a) A regular space $X$ is metrizable if and only if $X$ is collectionwise normal and developable $(=$ there is a sequence $\langle\Theta(n)\rangle$ of open covers of $X$ such that, for each $x \in X$, \{St $(x, \mathscr{G}(n)): n \in N\}$ is a local base at $x)$ [3];

(b) a completely regular space $X$ is developable if and only if $X$ is a $p$ space (cf. 4.3) and for each point $x \in X$ there is a sequence $\left\langle g_{n}(x)\right\rangle$ of open neighborboods of $x$ such that if $\left\langle x_{n}\right\rangle$ is a sequence in $X$ and if $y \in g_{n}\left(x_{n}\right)$ for each $n$, then $\left\langle x_{n}\right\rangle$ converges to $y, i_{0} e_{0}, X$ is developable if and only if $X$ is a semistratifiable p-space [12, Theorem 1.2].

6.3. Theorem. A space $X$ is metrizable if and only if $X$ is a monotonically normal p-space with a $G_{\delta}$-diagonal.

Conventions. In the following proof all closures are taken in $\beta X . H$ is assumed to be a function as described in Lemma 2.2(b) and if $p \neq q$ are points of $X$ we shall write $H(p, q)$ instead of $H(p,\{q\})$.

Proof. According to Theorem $3.1^{\circ}$, a monotonically normal space is collectionwise normal. Therefore, according to Theorem $6.2(a)$, it suffices to show that $X$ is developable. To accomplish this we use statement (b) of Theorem 6.2.

Because $X$ is a $p$-space with a $G_{\delta}$-diagonal, there is a sequence $\langle\mathcal{U}(n)\rangle$ of covers of $X$ by open subsets of $\beta X$ such that $\mathcal{U}(n+1)$ refines $\mathcal{U}(n)$ for each $n \in N$ and such that if $x \in X$, then $\bigcap\{$ St $(x, \mathcal{U}(n)): n \in N\}=\{x\}$. For each $y \in X$ and $n \in N$, let $D_{n}(y)$ be an open subset of $X$ such that (i) $y \in D_{n}(y)$ and (ii) $D_{n}(y)^{-}$is a subset of some member of $\mathcal{U}(n)$. Define $g_{n}(y)=H\left(y, X \backslash D_{n}(y)\right)$.

Suppose $\left\langle x_{n}\right\rangle$ is a sequence in $X$ such that $y \in g_{n}\left(x_{n}\right)$ for each $n \in N$. To show that $\left\langle x_{n}\right\rangle$ converges to $y$, it suffices to show that any subsequence $\left\langle x_{n_{k}}\right\rangle$ of $\left\langle x_{n}\right\rangle$, no term of which is $y$, has $y$ as its unique cluster point. Fix $k$. Because $x_{n_{k}} \neq y$, there is an integer $j_{k}$ such that if $j \geq j_{k}$, then $x_{n_{k}} \notin \operatorname{St}\left(y, \mathcal{U}\left(n_{j}\right)\right) \supset$ $D_{n_{j}}\left(x_{n_{j}}\right)$. If there were an integer $j \geq j_{k}$ such that $x_{n_{j}} \notin D_{n_{k}}\left(x_{n_{k}}\right)$, then it would follow that

$$
\begin{aligned}
g_{n_{j}}\left(x_{n_{j}}\right) & =H\left(x_{n_{j}}, X \backslash D_{n_{j}}\left(x_{n_{j}}\right)\right) \subset H\left(x_{n_{j}}, x_{n_{k}}\right) \subset X \backslash H\left(x_{n_{k}}, x_{n_{j}}\right) \\
& \subset X \backslash H\left(x_{n_{k}}, X \backslash D_{n_{k}}\left(x_{n_{k}}\right)\right)=X \backslash g_{n_{k}}\left(x_{n_{k}}\right),
\end{aligned}
$$

contradicting $y \in g_{n_{j}}\left(x_{n_{j}}\right) \cap g_{n_{k}}\left(x_{n_{k}}\right)$. Therefore, $x_{n_{j}} \in D_{n_{k}}\left(x_{n_{k}}\right)$ whenever $j \geq j_{k}$.

Because $\beta X$ is compact, the sequence $\left\langle x_{n_{k}}\right\rangle$ has a cluster point, say $z$, in $\beta X$. For each $k \in N, z \in\left\{x_{n_{j}}: j \geq j_{k}\right\}^{-} \subset D_{n_{k}}\left(x_{n_{k}}\right)^{-} \subset \mathrm{St}\left(y, \mathrm{U}\left(n_{k}\right)\right)$, the last inclusion being justified by conditions (i) and (ii) above, plus the fact that $y \in D_{n_{k}}\left(x_{n_{k}}\right)$. Therefore,

$$
z \in \bigcap\left\{\mathrm{St}_{\mathrm{t}}\left(y, \mathcal{U}\left(n_{k}\right)\right): k \in N\right\}=\{y\}
$$

as required. 
6.4. Remarks. (a) Theorem 6.3 generalizes a theorem in [2], attributed to E. Michael, which states that a stratifiable $p$-space is metrizable.

(b) Each of the hypotheses of Theorem 6.3 is necessary as can be seen from Examples 7.1 and 7.5.

(c) R. Gittings pointed out to the authors that the hypothesis of (6.3) that $X$ is a $p$-space can be replaced by the hypothesis that $X$ is an $M$-space [25], a $w \Delta$-space [6], or even a quasi-complete space in the sense of G. Creede [12].

\section{Examples.}

7.1. Example. There is a monotonically normal space $S$ which is hereditarily separable and hereditarily Lindelö (whence perfectly normal), which has a $G_{\delta}$-diagonal and which is not metrizable. Furthermore, the space $S \times S$ is not even normal. The space $S$ is the familiar Sorgenfrey line obtained by topologizing the set of real numbers so that sets of the form $[a, b[$ are basic open sets. That $S$ has the indicated properties follows from Corollary 5.6, since $S$ is clearly a generalized ordered space, and from the results of [26] and [20].

7.2. Example. There is a hereditarily paracompact, monotonically normal space $M$ which has a $G_{\delta}$-diagonal, and whose product with the usual space of irrationals is not normal. The space $M$ is the Michael line, obtained by topologizing the set of real numbers by taking all sets of the form $U \cup V$ to be open, where $U$ is open in the usual topology of the real numbers, and where $V$ is any set of irrational numbers. Clearly $M$ is a generalized ordered space; according to Corollary 5.6, $M$ is monotonically normal. The other properties of $M$ listed above are established in [23].

7.3. Example. There is a countable regular space which is not monotonically normal. In [15] it is proved that there is a countable regular space $X$ which is not stratifiable. (In fact, it was announced in [16] that $X$ can be taken to be an $\aleph_{0}$-space in the sense of [24].) Since any countable regular space is semistratifiable, it follows from Theorem 2.5 that $X$ is not monotonically normal.

7.4. Example. There are compact Hausdorff spaces which are not monotonically normal. Let $X$ be the countable regular space of Example 7.3. Since $X$ is not monotonically normal, neither is $Y=\beta X$ (cf. (2.3)(c)).

F. G. Slaughter, Jr., pointed out to the authors that any separable compact Hausdorff space is a continuous closed image of $\beta N$, the Stone-Cech compactification of the usual space of natural numbers. (Argument: Let $Y$ be separable, compact and Hausdorff. Let $f: N \rightarrow Y$ be any function whose image is a countable dense subset of $Y$. Then $\beta f: \beta N \rightarrow Y$ is necessarily a closed continuous onto map.) In particular, the space $Y=\beta X$ above is a continuous closed image of $\beta N$. Since $Y$ is not monotonically normal it follows from Proposition 2.7 that $\beta N$ cannot be monotonically normal. From this fact one can deduce that there is 
no nondegenerate space $Z$ such that $Z^{\mathfrak{m}}$ is monotonically normal for every cardinal number $m$ (or even for $m=2^{X_{0}}$ ).

7.5. Example. There is a compact homogeneous space $X$ which is first countable, hereditarily separable, perfectly normal and monotonically normal and yet which does not have a $G_{\delta}$-diagonal and is not metrizable. Let $X=$ $([0,1] \times\{0,1\}) \backslash\{(0,0),(1,1)\}$ have the usual open interval topology induced by the lexicographic order. It is easily seen that $X$ is a compact, homogeneous, nonmetrizable LOTS. Monotone normality of $X$ follows from Theorem 5.3 and the other listed properties of $X$ follow from the results of [21] since it is easily verified that $X$ is separable.

7.6. Example. There is a space $X$ such that $X^{n}$ (the product of $n$ copies of $X$ ) is monotonically normal for each $n \in N$ and yet $X^{\omega_{0}}$ is not monotonically normal. The points of the space $X$ are the ordinals which are less than or equal to the first uncountable ordinal $\omega_{1}$. We topologize $X$ in such a way that all countable ordinals are isolated points and basic neighborhoods of $\omega_{1}$ are sets of the form $\left.] a, \omega_{1}\right]$. Since $X$ is clearly a generalized ordered space, $X$ is monotonically normal according to (5.6). (It is interesting to note that, using a different order on $X, X$ is actually a LOTS.)

Fix $n \geq 1$ and let $Z=X^{n}$. For $1 \leq i \leq n$ and $z \in Z$ let $z_{i}$ denote the $i$ th coordinate of $z$. Let $E=\left\{z \in Z \mid z_{i}=\omega_{1}\right.$ for some $\left.i\right\}$ and for $z \in E$ let $E(z)=$ $\left\{i \mid 1 \leq i \leq n\right.$ and $\left.z_{i}=\omega_{1}\right\}$.

We verify that $Z$ admits a function $G(p, C)$ as described in (2.2)(b). Let $C \subset Z$ be closed and let $p \in Z \backslash C$. If $p \notin E$ then let $G(p, C)=\{p\}$. If $p \in E$ then there exist ordinals $u$ satisfying

(a) $\omega_{1}>u>\max \left\{p_{i} \mid i \notin E(p)\right\}$ (where we adopt the convention that $\max (\phi)=0)$ $z \backslash C$.

(b) $\left\{z \in Z \mid z_{i}=p_{i}\right.$ if $i \notin E(p)$ and $u<z_{i} \leq \omega_{1}$ if $\left.i \in E(p)\right\}$ is a subset of

(The sets described in (b) are simply basic neighborhoods of $p$ in the product topology of Z.) Define $u(p, C)$ to be the first ordinal satisfying both (a) and (b) and let

$$
G(p, C)=\left\{\dot{z} \in Z \mid z_{i}=p_{i} \text { if } i \notin E(p) \text { and } u(p, C)<z_{i} \leq \omega_{1} \text { if } i \in E(p)\right\} .
$$

That $G(p, C)$ satisfies conditions (i) and (ii) of Lemma 2.2(b) is immediate and it is a routine but tedious verification that condition (iii) is also satisfied. Thus $Z$ is monotonically normal.

It follows from Theorem 4.2 that the space $X^{\omega_{0}}$ is not monotonically normal because $X$ is not stratifiable: the singleton $\left\{\omega_{1}\right\}$ is a closed subset of $X$ which is not a $G_{\delta}$. 


\section{BIBLIOGRAPHY}

1. A. V. Arhangel'skiř, On a class of spaces containing all metric and all locally bicompact spaces, Dokl. Akad. Nauk SSSR 151 (1963), 751-754 = Soviet Math. Dokl. 4 (1963), 1051-1055. MR 27 \#2959.

2. - Mappings and spaces, Uspehi Mat. Nauk 21 (1966), no. 4 (130), 133184 = Russian Math. Surveys 21 (1966), no. 4, 115-162. MR 37 \#3534.

3. R. Bing, Metrization of topological spaces, Canad. J. Math. 3 (1951), 175-186. MR 13, 264.

4. G. Birkhoff, Lattice theory, 2nd rev. ed., Amer. Math. Soc. Colloq. Publ., vol. 15, Amer. Math. Soc., Providence, R. I., 1948. MR 10, 673.

5. C. J. R. Borges, On stratifiable spaces, Pacific J. Math. 17 (1966), 1-16. MR $32 \# 6409$.

6. —_ On metrizability of topological spaces, Canad. J. Math. 20 (1968), 795804. MR $37 \# 6910$.

7. — Elastic spaces are monotonically normal, Notices Amer. Math. Soc. 18 (1971), 840. Abstract \#71T-G158.

8. - Four generalizations of stratifiable spaces, Proc. Third Prague Topology Sympos. 1971 (to appear).

9. D. K. Burke and R. A. Stoltenberg, A note on p-spaces and Moore spaces, Pacific J. Math. 30 (1969), 601-608. MR $40 \# 3507$.

10. E. Čech, On bicompact spaces, Ann. of Math. (2) 38 (1937), 823-844.

11. - Topological spaces, Publ. House Czech Acad. Sci., Prague, 1966;

English transl., Wiley, New York, 1966. MR 35 \#2254.

12. G. D. Creede, Concerning semi-stratifiable spaces, Pacific J. Math. 32 (1970), 47-54. MR $40 \# 8006$.

13. Z. Frolik, On the topological product of paracompact spaces, Czechoslovak Math. J. 9 (84) (1959), 172-217. (Russian) MR 21 \#3821.

14. R. W. Heath, Semi-metrizable spaces and related topics, Topology Conference, Arizona State University, Tempe, Ariz., 1967, pp. 153-161.

15. - An easier proof that a certain countable space is not stratifiable, Proc. Washington State Univ. Conf. on General Topology (Pullman, Wash., 1970), Pi Mu Epsilon, Dept. of Math., Washington State Univ., Pullman, Wash., 1970, pp. 56-59. MR 42 \#1044.

16. - An $\mathrm{K}_{0}$-space which is not stratifiable, Notices Amer. Math. Soc. 17 (1970), 1040, Abstract \#679-G24.

17. R. W. Heath and D. J. Lutzer, A note on monotone normality, Notices Amer. Math. Soc. 18 (1971), 783. Abstract \#687-54-1.

18. —_ A characterization of monotone normality, Notices Amer. Math. Soc. 18 (1971), 1066. Abstract \#689-G8.

19. M. Katětov, Complete normatity of Cartesian products, Fund. Math. 35 (1948), 271-274. MR 10, 315 .

20. D. J. Lutzer, On generalized ordered spaces, Proc. Washington State Univ. Conf. on General Topology (Pullman, Wash., 1970), Pi Mu Epsilon, Dept. of Math., Washington State Univ., Pullman, Wash., 1970, pp. 102-110. MR $42 \# 1075$.

21. D. J. Lutzer and H. R. Bennett, Separability, the countable chain condition and the Lindelöf property in linearly orderable spaces, Proc. Amer. Math. Soc. 23 (1969), 664-667. MR $40 \# 2013$. 
22. M. J. Mansfield, Some generalizations of full normality, Trans. Amer. Math. Soc. 86 (1957), 489-505. MR 20 \#273.

23. E. Michael, The product of a normal space and a metric space need not be normal, Bull. Amer. Math. Soc. 69 (1963), 375-376. MR 27 \#2956.

24. - , Xospaces, J. Math. Mech. 15 (1966), 983-1002. MR 34 \#6723.

25. K. Morita, Products of normal spaces with metric spaces, Math. Ann. 154 (1964), 365-382. MR $29 \# 2773$.

26. R. H. Sorgenfrey, On the topological product of paracompact spaces, Bull. Amer. Math. Soc. 53 (1947), 631-632. MR 8, 594.

27. L. A. Steen, $A$ direct proof that a linearly ordered space is hereditarily collectionwise normal, Proc. Amer. Math. Soc. 24 (1970), 727-728. MR 41 \#2634.

28. L. B. Treybig, Concerning continuous images of compact ordered spaces, Proc. Amer. Math. Soc. 15 (1964), 866-871. MR 29 \#5218.

29. P. Zenor, Monotonically normal spaces, Notices Amer. Math. Soc. 17 (1970), 1034, Abstract \#679-G2.

DEPARTMENT OF MATHEMATICS, UNIVERSITY OF PITTSBURGH, PITTSBURGH, PENNSYLVANIA 15213 (Current address of R. D. Heath and D. J. Lutzer)

DEPARTMENT OF MATHEMATICS, AUBURN UNIVERSITY, AUBURN, ALABAMA 36830 (Current address of P. L. Zenor) 